\title{
The Overlapping Domain Decomposition Method for Exterior Harmonic Problem with Mixed Boundary Conditions
}

\author{
Quan Zheng and Wenjuan Shang* \\ College of Sciences, North China University of Technology, Beijing 100144, China \\ ${ }^{*}$ Corresponding author
}

\begin{abstract}
In this paper, a Schwarz alternating algorithm is proposed by introducing two artificial boundaries for exterior mixed boundary value problems of Laplace equation. The theorem of the geometrical convergence is obtained for the algorithm. Two numerical examples confirm the theoretical results and demonstrate the advantage in accuracy and efficiency.
\end{abstract}

Keywords-laplace equation; unbounded domain; mixed boundary conditions; domain decomposition

\section{INTRODUCTION}

Many physical phenomena develop elliptic problems in exterior regions, for examples, the flow of an incompressible irrotational fluid about a body and the study of electrostatics exterior to given surfaces are described by the Laplace equation (see, [1] and references therein). However, the unboundedness of the domains brings about the essential difficulty for solving these problems, and therefore, various methods known as coupling of BEM and FEM, the FEM with artificial boundary conditions and the domain decomposition method (DDM) had been constructed for the numerical solution of this kind of problems [2-4]. In particular, the DDM not only overcomes shortcoming of the stiffness matrix being not sparse banded in the coupling method, but allows the establishment of different mathematical models on different sub-regions and can achieve a high degree of parallel computing.

In this paper, we propose an overlapping DDM for solving the exterior Laplace equation with mixed boundary conditions, which is more general than the usually considered Dirichlet boundary condition. In Section 2, we prove the geometric convergence for the Schwarz alternating method. The accuracy and efficiency of this method is demonstrated in Section 3. Final remarks are given in Section 4.

\section{SCHWARZ Alternating ALgORITHM AND ITS CONVERGENCE}

Consider to solve the following exterior mixed boundary value problem of the Laplace equation:

$$
\begin{cases}\frac{\partial^{2} u}{\partial x^{2}}+\frac{\partial^{2} u}{\partial y^{2}}=0, & \text { in } \Omega, \\ u=u_{0^{\prime}} & \text { on } \Gamma_{D^{\prime}} \\ n_{x} \frac{\partial u}{\partial x}+n_{y} \frac{\partial u}{\partial y}=g, & \text { on } \Gamma_{N^{\prime}}\end{cases}
$$

where $\Gamma_{0}=\Gamma_{D} \cup \Gamma_{N}$ is a polygonal boundary surrounding the origin in $R^{2}, \Omega$ is the unbounded domain outside $\Gamma_{0}$, $u_{0} \in H^{1 / 2}\left(\Gamma_{D}\right), g \in H^{-1 / 2}\left(\Gamma_{N}\right)$, and $n=\left(n_{x}, n_{y}\right)$ is the outward unit normal vector on $\Gamma_{N}$. As long as $u$ is bounded at infinity, there is a unique solution $u$ of the problem (1),

$$
\begin{aligned}
& u \in W_{0}^{1}(\Omega) \text {, where } W_{0}^{1}(\Omega):=\left\{v \mid \frac{v}{\sqrt{x^{2}+y^{2}+1} \ln \left(x^{2}+y^{2}+2\right)},\right. \\
& \left.\frac{\partial v}{\partial x}, \frac{\partial v}{\partial y} \in L^{2}(\Omega)\right\} .
\end{aligned}
$$

Set bilinear form

$$
D(u, v)=\int_{\Omega} \nabla u \nabla v d x d y
$$

The corresponding variational form of (1) is

$$
\begin{cases}\text { Find } u \in V+u_{0}, & \text { such that } \\ D(u, v)=F(v), & \forall v \in V,\end{cases}
$$

where

$$
F(v)=\int_{\Gamma_{N}} g v d s, V=\left\{v\left|v \in W_{0}^{1}(\Omega), v\right|_{\Gamma_{D}}=0\right\} .
$$


Make a circle $\Gamma_{2}$, where dist $\left(\Gamma_{0}, \Gamma_{2}\right)>0$, such that $\Gamma_{0}$ is surrounded by $\Gamma_{2}$. Then make a closed circle $\Gamma_{1}$ which surrounds $\Gamma_{2}$. Let $\Omega_{1}$ denotes the annular domain between $\Gamma_{0}$ and $\Gamma_{1}$. Define $\Omega_{2}$ as the exterior unbounded domain with boundary $\Gamma_{2}$. Let

$$
\Omega_{11}=\Omega_{1} \cap\left(\bar{\Omega}_{2}\right)^{c}, \Omega_{12}=\Omega_{1} \cap \Omega_{2}, \Omega_{22}=\Omega_{2} \cap\left(\bar{\Omega}_{1}\right)^{c},
$$

where $\left(\bar{\Omega}_{i}\right)^{c}$ represents the complement of $\bar{\Omega}_{i}(i=1,2)$. Set $u_{i}^{(\mathrm{k})}=\left.u^{(\mathrm{k})}\right|_{\Omega_{i}}(i=1,2)$. Then we construct the following Schwarz alternating algorithm:

Step 1. Put any initial data $u_{2}^{(0)} \in H^{\frac{1}{2}}\left(\Gamma_{1}\right)$, for an example, $u_{2}^{(0)}=0$, and $\mathrm{k}:=1$.

Step 2. Solve a Mixed boundary value problem in bounded domain $\Omega_{1}$ :

$$
\begin{cases}\frac{\partial^{2} u_{1}^{(\mathrm{k})}}{\partial x^{2}}+\frac{\partial^{2} u_{1}^{(\mathrm{k})}}{\partial y^{2}}=0, & \text { in } \Omega_{1^{\prime}} \\ u_{1}^{(\mathrm{k})}=u_{0}, & \text { on } \Gamma_{D^{\prime}} \\ n_{x} \frac{\partial u_{1}^{(\mathrm{k})}}{\partial x}+n_{y} \frac{\partial u_{1}^{(\mathrm{k})}}{\partial y}=g, & \text { on } \Gamma_{N^{\prime}} \\ u_{1}^{(\mathrm{k})}=u_{2}^{(\mathrm{k}-1)}, & \text { on } \Gamma_{1} .\end{cases}
$$

Step 3. Solve a Dirichlet boundary value problem in unbounded domain $\Omega_{2}$ :

$$
\begin{cases}\frac{\partial^{2} u_{2}^{(\mathrm{k})}}{\partial x^{2}}+\frac{\partial^{2} u_{2}^{(\mathrm{k})}}{\partial y^{2}}=0, & \text { in } \Omega_{2}, \\ u_{2}^{(\mathrm{k})}=u_{1}^{(\mathrm{k})}, & \text { on } \Gamma_{2} .\end{cases}
$$

Step 4.

$e_{h}(k)=\max \left\{\sup _{\Gamma_{2}}\left|u_{1}^{(k)}-u_{1}^{(k-1)}\right|, \sup _{\Gamma_{1}}\left|u_{2}^{(k)}-u_{2}^{(k-1)}\right|\right\}$

Step 5. If $e_{h}(k)$ is small enough, stop; else turn to Step 2.
Extend $u_{1}^{(\mathrm{k})}$ and $u_{2}^{(\mathrm{k})}$ to $\Omega$ by $\left.u_{1}^{(\mathrm{k})}\right|_{\Omega_{22}}=u_{2}^{(\mathrm{k}-1)}$ and $\left.u_{2}^{(\mathrm{k})}\right|_{\Omega_{11}}=u_{1}^{(\mathrm{k})}$. Let spaces

$$
\begin{gathered}
V_{1}=\left\{v\left|v \in H^{1}\left(\Omega_{1}\right), v\right|_{\Gamma_{D}}=0,\left.v\right|_{\Gamma_{1}}=0\right\}, \\
V_{2}=\left\{v\left|v \in W_{0}^{1}\left(\Omega_{2}\right), v\right|_{\Gamma_{2}}=0\right\} .
\end{gathered}
$$

Then (3) is equivalent to the following variational problem:

$$
\left\{\begin{array}{l}
\text { Find } u_{1}^{(k)} \in V_{1}+u_{2}^{(k-1)}, \quad \text { such that } \\
D_{1}\left(u_{1}^{(k)}, v\right)=F(v), \quad \forall v \in V_{1},
\end{array}\right.
$$

(4) is equivalent to the following variational problem:

$$
\begin{cases}\text { Find } u_{2}^{(\mathrm{k})} \in V_{2}+u_{1}^{(k)}, & \text { such that } \\ D_{2}\left(u_{2}^{(k)}, v\right)=0, & \forall v \in V_{2},\end{cases}
$$

where

$$
\begin{gathered}
D_{i}(u, v)=\int_{\Omega_{i}} \nabla u \nabla v d x d y, i=1,2, \\
F(v)=\int_{\Gamma_{N}} g v d s .
\end{gathered}
$$

Then (3) and (4) are equivalent to the following variational problems:

$$
\left\{\begin{array}{l}
D_{1}\left(u_{1}^{(k)}-u, v_{1}\right)=0, \forall v_{1} \in V_{1}, \\
u_{1}^{(k)}-u_{2}^{(k-1)} \in V_{1},
\end{array}\right.
$$

and

$$
\left\{\begin{array}{l}
D_{2}\left(u_{2}^{(k)}-u, v_{2}\right)=0, \forall v_{2} \in V_{2}, \\
u_{2}^{(k)}-u_{1}^{(k)} \in V_{2} .
\end{array}\right.
$$

Let $P_{V_{i}}: V \rightarrow V_{i}(\mathrm{i}=1,2)$ denote the projection operators under the inner product $D_{i}(\cdot, \cdot)$ in $V$. Thus (7) and (8) are equivalent to

$$
D_{1}\left(u_{1}^{(k)}-u_{2}^{(k-1)}, v_{1}\right)=D_{1}\left(u-u_{2}^{(k-1)}, v_{1}\right), \quad \forall v_{1} \in V_{1},
$$


$D_{2}\left(u_{2}^{(k)}-u_{1}^{(k)}, v_{2}\right)=D_{2}\left(u-u_{1}^{(k)}, v_{2}\right), \quad \forall v_{2} \in V_{2}$.

We obtain

$$
\left\{\begin{array}{l}
u_{1}^{(k)}-u_{2}^{(k-1)}=P_{V_{1}}\left(u-u_{2}^{(k-1)}\right), \\
u_{2}^{(k)}-u_{1}^{(k)}=P_{V_{2}}\left(u-u_{1}^{(k)}\right),
\end{array} \quad k=1,2, \cdots\right.
$$

As $\quad u-u_{2}^{(k-1)}=u-u_{1}^{(k)}+u_{1}^{(k)}-u_{2}^{(k-1)}$ $u_{1}^{(k)}-u_{2}^{(k-1)} \in V_{1}, \quad u-u_{1}^{(\mathrm{k})} \in V_{1}^{\perp}$. Similarly, we have $u-u_{2}^{(\mathrm{k})} \in V_{2}^{\perp}$. Then (9) can be written as

$$
\left\{\begin{array}{l}
u-u_{1}^{(k)}=P_{V_{1}^{\perp}}\left(u-u_{2}^{(k-1)}\right), \\
u-u_{2}^{(k)}=P_{V_{2}^{\perp}}\left(u-u_{1}^{(k)}\right),
\end{array} \quad k=1,2, \cdots .\right.
$$

Let $e_{i}^{(k)}=u-u_{i}^{(k)}, i=1,2$, it follows

$$
\left\{\begin{array}{l}
e_{1}^{(k)}=P_{V_{1}^{\prime}} e_{2}^{(k-1)}, \\
e_{2}^{(k)}=P_{V_{2}^{\prime}} e_{1}^{(k)},
\end{array} \quad k=1,2, \cdots\right.
$$

This yields to

$$
\begin{cases}e_{1}^{(k+1)}=P_{V_{1}^{\perp}} P_{V_{2}^{\perp}} e_{1}^{(k)}, & k=1,2, \cdots \\ e_{2}^{(k+1)}=P_{V_{2}^{\perp}} P_{V_{1}^{\perp}} e_{2}^{(k)}, & k=0,1, \cdots\end{cases}
$$

This implies that, if $\left\{e_{i}^{(k)}\right\}, i=1,2$, are convergent, then their limits are in $V_{1}^{\perp} \cap V_{2}^{\perp}$.

Similar to the proofs of Theorem 1 in [5], the following results are obtained.

Lemma 1. Spaces $V, V_{1}$ and $V_{2}$ satisfy: $V=V_{1}+V_{2}$, $V_{1}^{\perp} \cap V_{2}^{\perp}=0$.

Lemma 2. If $V=V_{1}+V_{2}$, then for any $v \in V$ there exists a positive constant $C_{0}$ such that

$$
\begin{gathered}
\left(\left\|v_{1}\right\|_{1}^{2}+\left\|v_{2}\right\|_{1}^{2}\right)^{\frac{1}{2}} \leq C_{0}\|v\|_{1}, v=v_{1}+v_{2}, v_{1} \in V_{1}, v_{2} \in V_{2}, \\
\|v\|_{1} \leq C_{0}\left(\left\|P_{V_{1}} v\right\|_{1}^{2}+\left\|P_{V_{2}} v\right\|_{1}^{2}\right)^{\frac{1}{2}} .
\end{gathered}
$$

where $\|\cdot\|_{1, \Omega_{i}}$ denotes energy norm $\sqrt{D_{i}(\cdot, \cdot)}, i=(1,2)$.

Theorem 1. $\lim _{k \rightarrow \infty}\left\|e_{i}^{(k)}\right\|_{1}=0(i=1,2)$, and there exists a constant $\alpha \in[0.1)$, such that

$$
\left\|P_{V_{1}^{\perp}} P_{V_{2}^{\perp}}\right\| \leq \alpha,\left\|P_{V_{2}^{\perp}} P_{V_{1}^{\perp}}\right\| \leq \alpha,
$$

and

$$
\left\|e_{1}^{(\mathrm{k}+1)}\right\|_{1} \leq \alpha^{k}\left\|e_{1}^{(1)}\right\|_{1}, \quad\left\|e_{2}^{(\mathrm{k})}\right\| \leq \alpha^{k}\left\|e_{2}^{(0)}\right\|_{1}, \quad k=1,2, \cdots
$$

Theorem 1 shows that the above Schwarz alternating algorithm is geometrically convergent and the contraction factor is $\alpha$.

\section{NUMERICAL EXAMPLES}

Example 1. Consider to solve problem (1), where $\Gamma_{0}=\{(x, y)|| x|=1| y \mid, \leq 1\} \cup\{(x, y)|| x|\leq 1| y \mid,=1\}$, $\Gamma_{D}=\{(x, y)|x=1| y \mid, \leq 1\} \cup\{(x, y)|| x|\leq 1, \quad| y \mid=1\} \quad$, $\Gamma_{N}=\{(x, y)|x=-1| y \mid, \leq 1\}$, and the boundary conditions are obtained by the exact solution $u=\frac{x}{x^{2}+y^{2}}$.

The artificial boundaries $\Gamma_{1}$ and $\Gamma_{2}$ are circles with radiuses $R_{1}=4$ and $R_{2}=2$, respectively. The initial mesh $\Omega_{1 h}$ consists of uniform triangular partitions with 8 points on $\Gamma_{0}, \Gamma_{1}$ and $\Gamma_{2}$, respectively.

The finite element solution is obtained for problem (3) by linear FEM on $\Omega_{1 h}$. The numerical integral solution is obtained for problem (4) by composite trapezoidal formula on $\Gamma_{2} . e(k)$ denotes the maximal nodal error between the exact solution and iterative solution, $e_{h}(k)$ denotes the maximal nodal error of successive iterative solutions, and $q_{h}(k)$ represents the iterative convergence rate, i.e.,

$$
\begin{gathered}
e(k)=\max \left\{\sup _{\Gamma_{2 h}}\left|u-u_{1 h}^{(k)}\right|, \sup _{\Gamma_{1 h}}\left|u-u_{2 h}^{(k)}\right|\right\}, \\
e_{h}(k)=\max \left\{\sup _{\Gamma_{2 h}}\left|u_{1 h}^{(k-1)}-u_{1 h}^{(k)}\right| \sup _{\Gamma_{1 h}}\left|u_{2 h}^{(k-1)}-u_{2 h}^{(k)}\right|\right\}, \\
q_{h}(k)=e_{h}(k-1) / e_{h}(k) .
\end{gathered}
$$

The numerical results are given in Table 1 and Figure 1. 
TABLE I. THE RELATION BETWEEN CONVERGENCE RATE AND MESH

\begin{tabular}{|c|c|c|c|c|c|c|c|c|c|}
\hline $\mathrm{N}$ & meshes & $\mathrm{k}$ & 1 & 2 & 3 & 4 & 5 & 6 & 7 \\
\hline \multirow[t]{3}{*}{8} & $\mathrm{~h}$ & $e(k)$ & $1.7025 \mathrm{E}-1$ & $9.6678 \mathrm{E}-2$ & $8.3441 \mathrm{E}-2$ & $8.1248 \mathrm{E}-2$ & $8.0932 \mathrm{E}-2$ & $8.0906 \mathrm{E}-2$ & $8.0914 \mathrm{E}-2$ \\
\hline & & $e_{h}(k)$ & $3.9545 \mathrm{E}-1$ & $7.6610 \mathrm{E}-2$ & $1.3957 \mathrm{E}-2$ & $2.6150 \mathrm{E}-3$ & $5.2500 \mathrm{E}-4$ & $1.1900 \mathrm{E}-4$ & $3.1000 \mathrm{E}-5$ \\
\hline & & $q_{h}(k)$ & & 5.161879 & 5.489068 & 5.336852 & 4.980439 & 4.430905 & 3.780227 \\
\hline \multirow[t]{3}{*}{16} & $\mathrm{~h} / 2$ & $e(k)$ & $1.4098 \mathrm{E}-1$ & $5.8287 \mathrm{E}-2$ & $4.1711 \mathrm{E}-2$ & $3.8303 \mathrm{E}-2$ & $3.7539 \mathrm{E}-2$ & $3.7341 \mathrm{E}-2$ & $3.7280 \mathrm{E}-2$ \\
\hline & & $e_{h}(k)$ & $4.0531 \mathrm{E}-1$ & $8.2695 \mathrm{E}-2$ & $1.6576 \mathrm{E}-2$ & $3.4080 \mathrm{E}-3$ & $7.6400 \mathrm{E}-4$ & $1.9800 \mathrm{E}-4$ & $6.1000 \mathrm{E}-5$ \\
\hline & & $q_{h}(k)$ & & 4.901341 & 4.988825 & 4.863690 & 4.461336 & 3.861971 & 3.241780 \\
\hline \multirow[t]{3}{*}{32} & $\mathrm{~h} / 4$ & $e(k)$ & $1.2912 \mathrm{E}-1$ & $3.9048 \mathrm{E}-2$ & $1.9014 \mathrm{E}-2$ & $1.4164 \mathrm{E}-2$ & $1.2795 \mathrm{E}-2$ & $1.2333 \mathrm{E}-2$ & $1.2153 \mathrm{E}-2$ \\
\hline & & $e_{h}(k)$ & $4.0717 \mathrm{E}-1$ & $9.0069 \mathrm{E}-2$ & $2.0034 \mathrm{E}-2$ & $4.8490 \mathrm{E}-3$ & $1.3700 \mathrm{E}-3$ & 4.6200E-4 & $1.8000 \mathrm{E}-4$ \\
\hline & & $q_{h}(k)$ & & 4.520673 & 4.495819 & 4.131305 & 3.540690 & 2.965827 & 2.568918 \\
\hline \multirow[t]{3}{*}{64} & $\mathrm{~h} / 8$ & $e(k)$ & $1.2772 \mathrm{E}-1$ & $3.4223 \mathrm{E}-2$ & $1.2187 \mathrm{E}-2$ & $6.4080 \mathrm{E}-3$ & $4.6170 \mathrm{E}-3$ & $3.9530 \mathrm{E}-3$ & $3.6750 \mathrm{E}-3$ \\
\hline & & $e_{h}(k)$ & $4.0747 \mathrm{E}-1$ & $9.3498 \mathrm{E}-2$ & $2.2036 \mathrm{E}-2$ & $5.7890 \mathrm{E}-3$ & $1.8040 \mathrm{E}-3$ & $6.6800 \mathrm{E}-4$ & $2.8000 \mathrm{E}-4$ \\
\hline & & $q_{h}(k)$ & & 4.358076 & 4.243033 & 3.806701 & 3.208026 & 2.701405 & 2.388426 \\
\hline
\end{tabular}

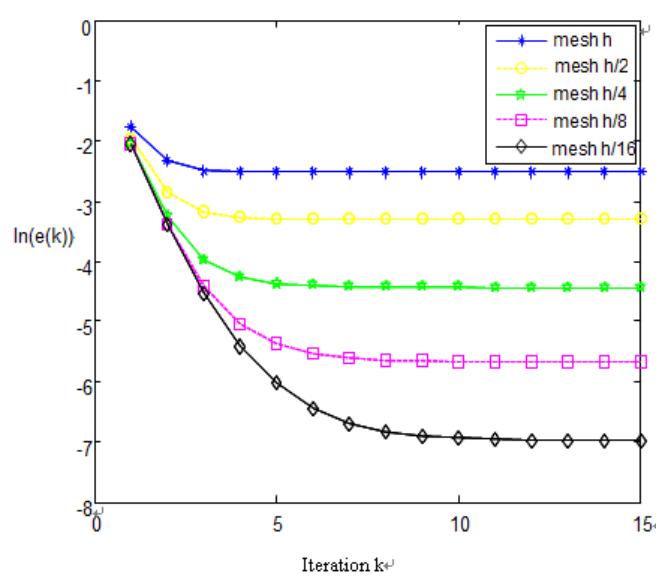

FIGURE I. THE RELATION BETWEEN RELATIVE ERRORS AND MESHES

The accurate finite element solution $u_{h}$ for problem (3) on $\Omega_{1 h}$ is obtained with exact boundary condition $\left.u\right|_{\Gamma_{1}}=\frac{1}{R_{1}} \cos \theta$. $e_{0}$ denotes the maximal nodal error $e_{0}=\sup _{\Gamma_{2 h}}\left|u-u_{h}\right|$. Take $e_{0}$ as a reference to examine the error $e(k)$, then, from another point of view, show the accuracy of iterative solution and the validity of the algorithm, see Table 1 and 2.

TABLE II. THE COMPARISON OF $e_{h}(10), e(10)$ AND $e_{0}$

\begin{tabular}{cccccc}
\hline & $e_{h}(10)$ & $e(10)$ & ratio & $e_{0}$ & ratio \\
\hline $\mathrm{h}$ & $1.0000 \mathrm{E}-6$ & $8.0925 \mathrm{E}-2$ & & $6.7550 \mathrm{E}-2$ & \\
$\mathrm{~h} / 2$ & $4.0000 \mathrm{E}-6$ & $3.7246 \mathrm{E}-2$ & 2.1727 & $2.8174 \mathrm{E}-2$ & 2.3976 \\
$\mathrm{~h} / 4$ & $1.5000 \mathrm{E}-5$ & $1.2027 \mathrm{E}-2$ & 3.0969 & $8.6720 \mathrm{E}-3$ & 3.2488 \\
$\mathrm{~h} / 8$ & $2.7000 \mathrm{E}-5$ & $3.4660 \mathrm{E}-3$ & 3.4700 & $2.4140 \mathrm{E}-3$ & 3.5924 \\
$\mathrm{~h} / 16$ & $3.7000 \mathrm{E}-5$ & $9.6500 \mathrm{E}-4$ & 3.5917 & $6.4000 \mathrm{E}-4$ & 3.7719 \\
\hline
\end{tabular}

The $q_{h}(k)$ in Table 1 shows that $e_{h}(k)$ decreases geometrically as the number of iterations $k$ increases and gradually tends to zero. The finer the mesh is, the smaller the error will be. The comparison between $e(10)$ and $e_{0}$ in Table 2 shows that the iterative solution is almost as accurate as the accurate finite element solution is. Although the accurate finite element solution is quadratically convergent with respect to $h$, it is a impractical solution. Moreover, Figure 1 shows that maximal errors with different meshes dwindle quickly until approach to stable states.

Example 2.To show the relation between convergence rate and overlapping degree, we compute on mesh $h / 4$ with different $R_{1}$ and $R_{2}$ in Example 1 .

TABLE III. THE RELATION BETWEEN CONVERGENCE RATE AND OVERLAPPING DEGREE

\begin{tabular}{ccccccc}
\hline$R_{2}$ & $R_{1}$ & $q_{h}(7)$ & $q_{h}(8)$ & $q_{h}(9)$ & $e_{h}(9)$ & $e(9)$ \\
\hline 2 & 4 & 2.5689 & 2.3526 & 2.2494 & $3.3970 \mathrm{E}-5$ & $1.2043 \mathrm{E}-2$ \\
2 & 8 & 3.4042 & 3.3464 & 3.3373 & $1.2586 \mathrm{E}-7$ & $3.5529 \mathrm{E}-2$ \\
4 & 8 & 1.9467 & 1.7193 & 1.6004 & $8.2031 \mathrm{E}-5$ & $1.4731 \mathrm{E}-2$ \\
2 & 12 & 3.9891 & 3.9906 & 3.9908 & $6.9561 \mathrm{E}-8$ & $6.6899 \mathrm{E}-2$ \\
\hline
\end{tabular}

Table 3 indicates that the larger the overlapping of subdomains is, the faster the convergence rate is.

\section{CONCLUSIONS}

In this work, we propose a Schwarz alternating algorithm for solving the Laplace equation with the mixed boundary conditions in the unbounded domain, and prove the corresponding geometric convergence. By means of this algorithm, a large scale multidimensional problem can be turned into two smaller discrete subproblems, which can reduce computing scale greatly. Moreover, its parallel computation in different subspaces can improve the efficiency. This algorithm gives a necessary complement to other methods in accordance with the feature of exterior problems. Two examples confirm the 
theoretical results and demonstrate the advantage in accuracy and efficiency.

\section{ACKNOWLEDGMENT}

This research is supported by Natural Science Foundation of China under the grant 11471019.

\section{REFERENCES}

[1] A. Bayliss, M. Gunzburger, and E. Turkel, "Boundary conditions for the numerical solution of elliptic equations in exterior regions," SIAM J. Appl. Math., vol. 42, no. 2, pp. 430-451, 1982.

[2] D. Givoli, Numerical Methods for Problems in Infinite Domains, Elsevier, Amsterdam, 1992.

[3] D. Yu, Natural Boundary Interal Method and Its Applications, Vol. 539, Kluwer Academic, Dordrecht, Netherlands, 2002.

[4] D. Han, X. Wu, Artificial Boundary Method, Tsinghua University Press, Beijing, 2012.

[5] D. Yu, "A domain decomposition method based on the natural boundary reduction over unbounded domain," Math. Numer.Sin., vol. 17, no. 1, pp. 448-459, 1994.

[6] J. Wu and D. Yu, "The overlapping domain decomposition method for harmonic equation over exterior three-dimensional domain," Comput.Math.., vol. 18, no. 1, pp. 83-94, 2000.

[7] F. Brezzi and C. Johnson, "On the coupling of boundary integral and finite element methods," Comput. Math., vol. 35, no. 151, pp. 10631079, 1980.

[8] K. Feng and D. Yu, "Canonical integral equations of elliptic boundary value problems and their numerical solutions," Proc. of China-France Symposium on Finite Element Method, Science Press, Beijing, pp. 211$252,1983$. 